\title{
Vibrotactile vigilance: The effects of costs and values on signals
}

\begin{abstract}
Vibrotactile vigilance behavior was examined for the effects on signal detection performance of placing costs on misses and false detections and a positive value on the correct detection of a signal. Analyses over time showing that the frequencies of correct detection and false detection of signals decreased, while response times increased, indicated that increased costs for misses and false alarms lead to poorer correct detection performance, while the value placed on the correct detection of a signal had little effect. The d' statistic of signal detection theory was invariant with both signal costs and time, while $\beta$ varied with both factors. These results imply that the performance decrement during a vigil was due to an increased strictness in the criterion $(\beta)$ the S set for deciding whether or not a signal was present. The cost factors were effective in manipulating performance during the watch by causing changes in the S's decision criteria. Findings from this study clearly support those predicted by the "Decision Theory" of Swets, Tanner, and Birdsall (1961); this is suggested as perhaps being the best available basis for a theory of vigilance at this time.
\end{abstract}

Values and costs placed on correct and incorrect performance in the vigilance task are variables which have received little research attention. However, when the vigilance task is viewed in terms of the Tanner and Swets (1954) and Swets, Tanner, and Birdsall (1961) signal detection theory model, such variables affecting the observer's decision processes become critical.

Payoff matrices have been constructed in studies of signal detection, but generally these do not isolate the independent contributions made by the values and costs placed on correct and incorrect performance. Little previous research has been given to the effect of target value on performance. Bergum and Lehr (1964) report a facilitatory effect for monetary incentives on vigilance performance, but it was only a short-term one. Levine (1966) found that signal value did not affect auditory vigilance performance. In studying costs he found that increasing them for false detections and misses led to poorer detection performance.

Different reasons have been suggested for the performance decrement commonly observed in vigilance tasks. Signal detection theory suggests that performance decrement during a watch may be due to a shift in the criterion level set by an observer, or to a change in the underlying efficiency of his senses. Egan, Greenberg, and Schulman (1961) suggested there is no real decrement in operator efficiency on vigilance tasks; rather there is a progressive change towards greater caution in the observer's criterion. Broadbent and Gregory (1963), Levine (1966) and Davenport (1968) support this view for auditory vigilance tasks, in each case $\beta$ increasing with time on watch, while $d^{\prime}$ remained constant for all levels of caution. However, in a visual vigilance situation Mackworth and Taylor (1963) found that $\log \mathrm{d}^{\prime}$ decreased significantly with time on watch. Mackworth (1964, 1965) found further support for this argument. Loeb and Binford (1964) found d' invariant with time in an auditory vigilance task, Ss being required to detect $62-\mathrm{dB}$ tones among a series of $60-\mathrm{dB}$ tones. More recent studies by Broadbent and Gregory (1965) using a visual vigilance task essentially replicated the findings of Loeb and
Binford (1964) and Broadbent and Gregory (1963). Apparently then the discrepant results of Mackworth (1964, 1965) and Mackworth and Taylor (1963) cannot be accounted for simply by differing sensory modalities.

The theory of signal detectability (TSD) considers detection performance as a judgment process in which stimuli are classed by the observer as signals or nonsignals (noise) depending on the criterion he adopts. The criterion is a cutoff on a mathematical transformation of two overlapping discriminal dispersions, in Thurstone's (1927) sense, which represent signal and nonsignal stimulation. The theory assumes that the signal and the nonsignal dispersions are both Gaussian and equal. Although it appears possible that these assumptions could be violated in a vigilance task, the potential of Signal Detection Theory for research into vigilance tasks appears to make its application a justifiable one in the present study.

The present study is unique in its examination of the effect of values and costs on correct and incorrect performance for a cutaneous vigilance task. Vigilance is regarded in terms of statistical decision theory. Manipulation of decision criteria in terms of costs of misses and false detections and values of correct detections (hits) would be expected to leave d' unaltered while $\beta$ increased with time. Thus the observer would adopt a more cautious criterion as the watch proceeded. It was hypothesized that this increase in $\beta$, if observed, would parallel a performance decrement during the watch, thus providing a possible explanation for its occurrence. Finally, it was hypothesized that the cost factors placed on signals during the watch would effectively manipulate the observer's detection performance by altering the frequencies of correct and incorrect detections obtained by each value group.

\section{Subjects}

\section{METHOD}

The Ss were 15 male and 15 female students of the University of Newcastle.

\section{Procedure}

Signals were transmitted to a modified Sherrick transducer attached to the left volar forearm of the S (Sherrick, 1965). The transducer was cylindrical rather than cubical in form. Each S's cutaneous threshold was found using three runs of the method of limits technique from above and below the threshold value. The range of thresholds so found was small. During threshold testing the vibration was present for $1-\mathrm{sec}$ periods at 6 -sec intervals. The power supplied to the vibrator could be varied in steps of $0.1 \mathrm{~V}$ using a variable ratio transformer.

During the $75-\mathrm{min}$ watch, which unknown to the $S$ was divided into three identical 25 -min periods, the vibrationary stimulus of $1-\mathrm{sec}$ duration and $1 \mathrm{~V}$ above threshold intensity was presented, the $S$ receiving 10 signals during each of the three 25 -min sections of the watch. Intersignal intervals were $95,29,299,73,168,303,201,55,37$, and $240 \mathrm{sec}$.

The values of correct and incorrect performance were manipulated by awarding or subtracting points. Ss were randomly allocated to Groups 1-5 as shown in Table 1. Groups $1,2,3$, and 4 represent a 2 by 2 factorial design with costs of misses and false detections as parameters. Groups 1-5 
Table 1

Costs in Points of False Detections and Misses and Values of Correct Detection (Hits) for Each Experimental Group

\begin{tabular}{cccc}
\hline Group & Cost of False Detection & Cost of Miss & Value of Hit \\
\hline 1 & 1 & 1 & 0 \\
2 & 8 & 8 & 0 \\
3 & 1 & 8 & 0 \\
4 & 8 & 1 & 0 \\
5 & 0 & 0 & $1,4,16$ \\
\hline
\end{tabular}

contained three male and three female Ss; for Group 5, the value of correct responses to signals in each of the three identical 25-min sections of the watch was 1,4 , or 16 points. As there were three equal time periods in each watch and three signal values $(1,4$, or 16 points), there were six possible permutations of a particular signal value across periods of the watch. One $\mathrm{S}$ was randomly assigned to each of these six possible permutations. This procedure controlled for the possibility that performance might be differentially affected by an interaction between time on watch and signal value.

During the instruction period, before the watch, the $S$ was given a verbal description of the task and a card showing the costs set on a false detection or a miss and the value of a correct detection. He was instructed to study the values and costs placed on the signals and give his best possible performance. He was informed that the shortest intersignal interval would be at least $25 \mathrm{sec}$ and that only one signal could be present in a $25-\mathrm{sec}$ interval. This interval was measured by a clockhand visible to the $S$ which moved through 360 deg once every 25 sec. a $10-$ min practice watch followed in which four signals were inserted, each of $1-\mathrm{sec}$ duration and $1 \mathrm{~V}$ above the particular S's threshold. The S was then told how well he had done. The experiment took place in a quiet laboratory, the $S$ being visible through a one-way screen.

\section{RESULTS}

An analysis of variance ( $\mathrm{AOV}$ ) was computed for each of the three dependent variables: (1) number of correct detections, (2) number of false detections, and (3) response latency.

This analysis employed Groups 1, 2, 3, and 4, thereby allowing for independent estimates of costs for false detections and misses. The AOV used was as described by Winer (1962, pp. 298-318) as a two-factor experiment with repeated measures for the time-on-watch factor. Results for each of the dependent variables are discussed separately below. A separate analysis was computed to assess the effect of signal value on signal detection performance. This was as described by Winer (1962, pp. 105-139).

Table 2

Frequencies of Correct Detections and False Detections for Groups 1-4 and for the Three Signal Values of Group 5 Over the Three Equal Time Intervals of the Watch

\begin{tabular}{llcc}
\hline Group & Interval 1 & Interval 2 & Interval 3 \\
\hline 1 & $47^{*} ; 12^{* *}$ & $40 ; 8$ & $35 ; 5$ \\
2 & $34 ; 5$ & $33 ; 4$ & $28 ; 3$ \\
3 & $43 ; 9$ & $36 ; 6$ & $33 ; 5$ \\
4 & $40 ; 7$ & $36 ; 6$ & $30 ; 4$ \\
$5(1)$ & $18 ; 9$ & $17 ; 5$ & $15 ; 3$ \\
$5(4)$ & $15 ; 3$ & $18 ; 6$ & $16 ; 4$ \\
$5(16)$ & $18 ; 7$ & $15 ; 4$ & $16 ; 5$ \\
\hline
\end{tabular}

*Frequency of Correct Detections

**Frequency of False Detections

(I), (4), (16)-The three values placed on correct responses to signals for Group 5.

\section{Number of Correct Detections}

The AOV of number of correct detections indicated significant effects due to Time Intervals, $F(2,40)=22.51$, $p<.01$. The effect of costs for misses for this dependent variable was insignificant as was the interaction between value groups and time intervals. The effect of costs for False Detections approached significance $(F(1,20)=3.95, p>.05$ but did not reach it. Levine (1966) in an auditory vigilance situation found significant effects due to the costs of misses and false detections as well as blocks of trials. This difference in the factors found significant in Levine's (1966) study and the present one appears interesting.

Signal detection apparently was not affected by the positive payoffs placed on correct detections, as shown in Table 2 by the almost equal numbers of correct detections at the three levels of signal value, $F(2,10)=1.58, p>.05$.

\section{Number of False Detections}

This AOV showed significant effects for the cost of a False Detection, $F(1,20)=10.05, p<.01$ and over Time Intervals, $F(2,40)=8.80, p<.01$. The cost of a miss approached significance, $F(1,20)=4.09, p>.05$ but the interaction between Value Groups and Time Intervals was insignificant. The cost of a False Detection appeared to be more potent than the cost of a miss, this trend appearing in the frequencies of correct detections and false detections given by Groups 1-4 as shown in Table 2. The AOV for Group 5 showed the number of False Detections at all levels of signal value to be almost equal, $\mathrm{F}(2,10)=0.11, \mathrm{p}>.05$.

\section{Response Latency}

For the latency data detection times were significantly increased by the cost of a miss, $F(1,20)=10.45, p<.01$ and over Time Intervals, $F(2,40)=12.03, p<.01$. The cost of a False Detection was insignificant as was the interaction between different value groups and time intervals. The three-signal values placed on correct detection performance had no significant differing effect on response latency, $F(2,10)=1.18, p>.05$. These results appear to demonstrate more efficient performance by $\mathrm{Ss}$ in the early stages of the watch, while the usual performance decrement later became more noticeable. Table 3 contains the means and standard deviations of response times for each of the Groups 1-5 over the entire period of the watch. Response times were only determined for correct detections, the $\mathrm{S}$ responding as soon as possible after the detection.

Table 3

Means and Standard Deviations of Response Times for Groups 1-5

\begin{tabular}{ccc}
\hline Group & Mean Response Time (sec) & Standard Deviation \\
\hline 1 & 1.13 & .12 \\
2 & 1.24 & .10 \\
3 & 1.19 & .08 \\
4 & 1.15 & .11 \\
5 & 1.07 & .07 \\
\hline
\end{tabular}

\section{Statistical Decision Theory}

The $\mathrm{d}^{\prime}$ and $\beta$ statistics of statistical decision theory were calculated from observed proportions of correct and false detections, assuming equal variances of the noise and signal + noise distributions. The data were pooled across Ss in each of the Groups $1-5$ to increase the number of observations available and thus obtain more stable estimates of $d^{\prime}$ and $\beta$. This pooling procedure was considered reasonable as the number of false detections was about equal for all Ss. An estimate of the false alarm rate was obtained by telling Ss that only one signal could occur in each 25 -sec interval and 
Table 4

Values of $\beta$ and $d^{\prime}$ for Groups 1-5 Over the Three Equal Time Intervals of the Watch

\begin{tabular}{clrr}
\hline Group & Interval 1 & \multicolumn{1}{c}{ Interval 2} & Interval 3 \\
\hline 1 & $3.41^{*} ; 2.52^{* *}$ & $5.87 ; 2.38$ & $9.45 ; 2.35$ \\
2 & $9.52 ; 2.32$ & $11.67 ; 2.36$ & $15.06 ; 2.24$ \\
3 & $4.98 ; 2.44$ & $7.92 ; 2.30$ & $9.58 ; 2.27$ \\
4 & $6.60 ; 2.49$ & $7.92 ; 2.30$ & $11.77 ; 2.26$ \\
5 & $1.88 ; 2.56$ & $2.40 ; 2.59$ & $3.41 ; 2.53$ \\
\hline
\end{tabular}

*Values of $\beta$

**Values of $d^{*}$

providing a measure of this interval in the form of a pointer moving through $360 \mathrm{deg}$ once every $25 \mathrm{sec}$. This 25 -sec interval was less than the shortest intersignal interval. d' was invariant with time, different costs for false detections and misses, and different values for signals. $\beta$ increased with time as shown in Table 4 demonstrating a trend of increasing strictness of the decision criteria. $\beta$ was also affected by the varying costs and values placed on S's performance, being larger for groups working under high costs than those under low costs as shown in Table 4, this relationship being maintained over time.

\section{Number of Correct Detections}

\section{DISCUSSION}

There was a progressive and significant decrement in the number of correct detections. The significance of the cost of a false detection indicated that high costs of false detections resulted in a reduced number of correct detections; this is consistent with a decision-making view of detection, where higher costs for false detections would result in more caution in responding and therefore decrease the number of correct detections. The results for signal value over the three dependent variables appear to show clearly that values had no effect on results but costs placed on false detections and misses did affect the results. Between the two independent negative payoffs, costs for false detections was the more effective, this finding being consistent with the small numbers of false detections given by all Ss, and by Ss' verbal reports that they were more concerned with avoiding errors of commission than errors of omission. False detections became significantly less frequent in each of the Groups 1-4 with increasing time over the three equal periods of the watch.

\section{Response Latency}

The absolute response times during the three periods of the watch were quite high $(1,000-1,500 \mathrm{msec})$; this lends further support to the detection of signals in the present vigilance situation as being a decision process. Response times for pilot Ss working without payoff matrices were much shorter (e.g., $800 \mathrm{msec}$ ). The response latency times increased significantly for each of Groups 1-4 over the three equal periods of the watch.

\section{Statistical Decision Theory}

Statistical decision theory predicted that $\mathrm{d}^{\prime}$ would be invariant with costs and values placed on signals. This was verified, $d^{\prime}$ also being invariant with time. $\beta$ was affected both by time and the costs and values given to signals. The increase in $\beta$ with time intervals was an increase in the strictness of the S's criterion of what constitutes a signal. This would result in fewer correct and false detections with time according to statistical decision theory. This prediction was verified in this study. Cost factors were effective in manipulating the Ss' decision criteria, for as shown in Table $4, \beta$ was larger for groups working under high costs than those working under low costs. Considering some of the values of $\beta$ shown in Table 4 perhaps they should not be thought of as a simple index of criterion, but rather as showing that important assumptions of the theory of statistical decision (TSD) were probably not met. Green (1960) indicates that a $\beta=5.0$ would be extreme in a psychophysical detection experiment, but in the present study $\beta$ rises to 15.06 . These high values might be explained by the Jerison, Pickett, and Stenson (1965) approach of assuming different methods of observing at various times during the watch. In the vigilance situation, as opposed to the psychophysical experiment for which TSD measures are appropriate, the observer is under no constraint to maintain a consistent observing mode, his observing behavior being externally paced. The methods of observing employed during a vigilance watch as suggested by Jerison et al (1965) were: (a) Alert observing-this being the optimum observing technique of the psychophysical experiment, (b) blurred observing-a process which increased the variance of both the $\mathrm{N}$ and $\mathrm{S}+\mathrm{N}$ distributions relative to that of alert observing, and (c) distraction-observing which includes daydreaming or autistic behavior occurring during a prolonged vigil. Here $\beta$ might increase greatly, being so severe that no stimuli would be reported either as signals or false alarms. Thus, perhaps a better interpretation of some of the results in Table 4 would be that the observers mixed their observing methods, rather than accept the high $\beta$ values as extremely cautious criteria in TSD terms.

The present study supports the hypothesis of Egan et al (1961) that there is a progressive change in the observer's criterion, becoming more conservative in response as the watch proceeds. There appears to be no evidence for a significant decrement in operator sensitivity during the watch, the performance decrement, under all dependent variables, apparently being due to a change in the observer's criterion as the watch proceeds. This study indicates that costs for misses and particularly false detections markedly affect performance on a vigilance task; the findings for the cause of the performance decrement support previous work by Broadbent and Gregory (1963, 1965), Loeb and Binford (1964) and Levine (1966). Further research to account for the discrepant results of Mackworth (1964, 1965) and Mackworth and Taylor (1963) seems warranted. These results are very similar to those obtained in a previous study on auditory vigilance (Davenport, 1968). This similarity of results for the two senses, auditory and cutaneous, appears as a promising sign for future studies. Perhaps a variety of vigilance tasks over a number of sensory modalities is required to solve this dilemma. Since results in this study all clearly support Swets et al (1961) "Decision Theory," this theory appears to be the most promising basis available for a theory of vigilance.

\section{REFERENCES}

BERGUM, B. O., \& LEHR, D. J. Monetary incentives and vigilance. Journal of Experimental Psychology, 1964, 67, 197-198.

BROADBENT, D. E., \& GREGORY, M. Vigilance considered as statistical decision. British Journal of Psychology, 1963, 54, 309-323,

BROADBENT, D. E., \& GREGORY, M. Effects of noise and of signal rate upon vigilance analysed by means of decision theory. Human Factors, 1965, 7, 155-162.

DAVENPORT, W. G. Auditory vigilance: The effects of costs and values on signals. Australian Journal of Psychology, 1968, 20, in press.

EGAN, J. P., GREENBERG, G. Z., \& SCHULMAN, A. I. Operating characteristics, signal detectability, and the method of free response. Journal of the Acoustical Society of America, 1961, 33, 993-1007.

GREEN, D. M. Psychoacoustics and detection theory. Journal of the Acoustical Society of America, 1960, 32, 1198-1203.

JERISON, H. J., PICKETT, R. M., \& STENSON, H. H. The elicited observing fate and decision processes in vigilance. Human Factors, $1965,7,107-128$

LEVINE, J. M. The effects of values and costs on the detection and identification of signals in auditory vigilance. Human Factors, 1966, 8 , 525-537. 
LOEB, M., \& BINFORD, J. R. Vigilance for auditory intensity changes as a function of preliminary feedback and confidence level. Human Factors, 1964, 6, 445-458.

MACKWORTH, J. The effect of true and false knowledge of results on detection of signals in a vigila.ce task. Canadian Journal of Psychology, 1964, 18, 106-117.

MACKWORTH, J. Decision interval and signal detectability in a vigilance task. Canadian Journal of Psychology, 1965, 19, 111-117.

MACKWORTH, J. \& TAYLOR, M. M. The $\mathrm{d}^{\prime}$ measure of signal detection in vigilance-like situations. Canadian Journal of Psychology, 1963.17. 302-311.

SHERRICK, C. E. Simple electromechanical vibration transducer. Review of Scientific Instruments, 1965, 36, 1893-1894.

SWETS, J. A., TANNER, W. P., \& BIRDSALL, T. G. Decision processes in perception. Psychological Review, 1961, 68, 301-340.
TANNER, W. P., \& SWETS, J. A. A decision making theory of visual detection. Psychological Review, 1954, 61, 401-409.

THURSTONE, L. L. Psychophysical analysis. American Journal of Psychology, 1927, 38, 368-389.

WINER, B. J. Statistical principles in experimental design. New York: McGraw-Hill, 1962.

\section{NOTE}

1. Address: Department of Psychology, University of Newcastle, N.S.W., Australia.

(Accepted for publication September 11, 1968.) 